\title{
Téoros
}

Revue de recherche en tourisme

\section{Le blues du businessman}

\section{Jean Pelletier}

Volume 15, numéro 3, automne 1996

Le tourisme d'affaires

URI : https://id.erudit.org/iderudit/1075060ar

DOI : https://doi.org/10.7202/1075060ar

Aller au sommaire du numéro

Éditeur(s)

Université du Québec à Montréal

ISSN

0712-8657 (imprimé)

1923-2705 (numérique)

Découvrir la revue

Citer cet article

Pelletier, J. (1996). Le blues du businessman. Téoros, 15(3), 13-19.

https://doi.org/10.7202/1075060ar

Ce document est protégé par la loi sur le droit d'auteur. L'utilisation des services d'Érudit (y compris la reproduction) est assujettie à sa politique d'utilisation que vous pouvez consulter en ligne.

https://apropos.erudit.org/fr/usagers/politique-dutilisation/
Cet article est diffusé et préservé par Érudit.

Érudit est un consortium interuniversitaire sans but lucratif composé de l’Université de Montréal, l'Université Laval et l'Université du Québec à Montréal. Il a pour mission la promotion et la valorisation de la recherche. https://www.erudit.org/fr/ 


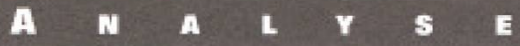

\section{LE BLUES DU BUSINESSMAN}

Jean Pelletier, président directeur général

Centre d'études du tourisme et consultant en communication

Chaque jour des dizaines de millions de gens d'affaires - hommes et, de plus en plus, femmes - arrivent (sowent à la course) en taxi (ou en limousine) à l'un des quelque 6000 aéroports du monde pour sauter dans un avion conmercial (ou un jet corporatif) ou parfois tout simplement, participer à une réunion avec un autre voyageur de passage ou en quasi transit ${ }^{\text {in }}$.

Comme l'exprime très bien un fort en thème de la prospective * Travel is and will continue to be the world's largest industry. No matter how sophisticated the telecommunications infrastructure becomes, or how many of our business or leisure activities can be conducted from the comfort of our living rooms, most of us will continue to get up out of our easy chairs because there is no substitute for the real thing. $w^{121}$ Chose certaine - croissance démographique galopante à l'appui - les industriels (et les gouvernements) des vingt-cinq prochaines années vont vendre des dizaines, voire des centaines

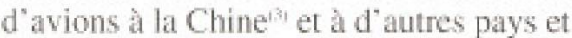
entreprises, et presque toutes les autorités locales (publiques et privées) vont devoir agrandir ou construire des aéroports, amenager des aérogares, doubler et allonger des pistes et... penser aux accès des aéroports pour pouvoir accéder aux avions (ou... aux salles de réunions des hôtels du voisinage). Plus que jamaís l'anticipation semble nécessaire et déterminante, même si nous pouvons avancer peu de précisions car rien n'est fiable, pour paraphraser l'observatrice Françoise Giroud.
L'attaché-case à la main, le sac Louis Vuitton (ou son imitation) en bandoulière et tirant une mini valise sur roulettes ${ }^{4 t}$ ? que le Pdg, la cadre supérieure ou le haut fonctionnaire peut apporter en cabine, sont les attributs du voyageur d'affaires qui voit à ses affaires et à ses apparences.

À l'heure où l'on \& parle (...) de l'internationalisation de tout et de la globalisation de rien $w^{(5)}$, à l'ère des villes-régions qui attirent les entreprises à haut contenu de savoir, exportent et rayonnent aux quatre coins du monde ${ }^{163}$, dans un contexte de déréglementation et de libéralisation du ciel (en Amérique du Nord, en Europe et, bientôt, pour le transport aérien transatlantique ${ }^{07}$ ), plus de gens (d'affaires) voyagent plus souvent plus ou moins loin.

Il y a maintenant des avions de tous les gabarits qui permettent aux transporteurs de $s^{*}$ adapter aux marchés de centaines de paires de villes, d'augmenter les liaisons directes (souvent sans escale) et, concurrence aidant, de maintenir le prix des billlets à un niveau, somme toute, peu élevé.
$\mathrm{D}^{+}$autant que les entreprises (compagnies aériennes avec, comme partenaires, les chaînes hôtelières) ont, au fil des dix dernières années, multiplié les mesures incitatives et les programmes de reconnaissance pour augmenter leur achalandage ou... conserver leurs clients. De puissantes stratégies marketing viennent appuyer les investissements considérables' (acquisition d'avions, renouvellement de flottes, aménagements intérieurs avec équipements sophistiqués) pour encourager les gens à emprunter le meilleur des transporteurs. aux dires de chacune des cinquante compagnies aé. riennes les plus connues'i"'.

Jadis, chaque pays voulait afficher ses couleurs au-dessus des nuages. Ce sont maintenant les agglomérations urbaines marchés (et leurs gens d'affaires) qui veulent avoir des liaisons efficaces avec les autres villes du monde, au grand plaisir des constructeurs (Airbus, Boeing et... Bombardier), et qui doivent prévoir des conditions facilitantes, au premier chef, des aćroports, un comme à Toronto et vancouver ou plusieurs comme à Londres et New York.

\section{DES PORTS AUX AÉROPORTS}

LLa vitalité d'une nation se mesure à l'activité de ses portss, écrivalit, voici tout juste un siècle, le Danois Knut Thorensen. Ce constat vaut désormais pour les aéroports. devenus les véritables baromètres de l'économie (d'un pays, d'une région, d'une métropole), autant que de l'état de ses relations internationales. Jamais auparavant, lạ planète n'a été si couverte de chantiers aéroportuaires, dont 
certains - asiatiques - de grande taille. On agrandit, on adapte, on crese.

En 1997, pendant que les Canadiens pourront emprunter le nouveau Pont de la Conféderation entre l'Île-du-PrinceEdouard et le Nouveau-Brunswick, pendant que les Britanniques tarderont encore à construire des voies d'accès fonctionnelles pour rentabiliser l'Eurotunnel (et protéger les compagnies de ferries trans-Manche), pendant que les Québécois des régions vont redécouvrir les turbo-propulsés d'InterCanadien, la Chine continuera de s"ouvrir au monde avec la mise en service du nouvel aéroport de Hong Kong (Chep Lak Kok) aménagé sur une île entièrement remodelếe et agrandie d'environ 1000 hectares gagnés sur la mer. La plate-forme devrait accueillir, dans un premier temps, environ 35 millions de passagers par année, 70 avions à l'heure, 24 heures sur 24 ; $y$ débarqueront des investisseurs, ingénieurs, techniciens, artistes et enseignants du... Québec 'll!

Airbus et Boeing sont d'accord: le moteur de croissance, c"est l"Asie"12. D'ici l'an 2000, la Chine aura construit ou modernise 100 aéroports. Et lia Corée! Et le Japon avec, notamment, la plateforme de Kansai construite dans la baie d'Osaka! Sans oublier l'Inde: les gens d'affaires de cet autre géant, démographiquement parlant, représentent un bassin de voyageurs intemationaux aussi important que la population totale du Québec... 13! Mêmé Singapour, avec moins de trois millions d'habitants peut compter sur deux transporteurs aériens mondialement réputés.

En Europe, à Francfort par exemple, une seconde gare fetroviaire, destinée aux trains à grande vitesse, devrait voir le jour au cours des prochaines années. Le nouveau terminal de l'aéroport de Bruxelles. capitale du continent, constitue le plus important projet mené par la Belgique en cette fin de siecle. Cet automne, on a decidé du périmètre du site d'un futur troisième aéroport parisien à... $150 \mathrm{~km}$ de la capitale (Eure-et-Loire). Quant à Heathrow, le plus important des quatre ároports londoniens (avec Gatwick, Luton, Stansted), on y a inauguré un Fligh Connections Center (FCC) pour les quelque sept millions de passagers annuels en... transit, etc. De Rome à Zurich des chantiers sont annoncés ${ }^{i+1}$.
Plus pres de nous, New York, avec 75 millions de passagers, représente le premier complexe aéroportuaire du monde (J.F. Kennedy, La Guardia et Newark). Montreal et Toronto sont bien arrimés à cette plaque tournante. Les gens de lat capitale québécoise doivent passer par Montréal pour s'y rendre!!

Par ailleurs, ceux et celles qui veulent savoir à quoi pourrait ressembler l'aéroport du XXle siecle peuvent aller au Colorado, dans la ville d'adoption des Nordiques et de Patrick Roy : le Denver International Airport est le seul aéroport entièrement nouveau construit au cours des vingt dernières années aux EtatsUnis, Le chantier a téte depuis 1982, une aventure couteuse. La plate-forme fait deux fois et demie Paris intra-muros ou deux fois Manhattan. Bienvenue au monde entier... venant d'Asie ou d'ailleurs pour les affaires et... le ski. Baptisế South Service Center, la zone de services du DLA a été qualifiée de ville dans la ville par ses concepteurs.

Plusieurs, plus que l'on a coutume de le penser, prement l'avion comme d'autres prennent le train de banlieuc. Entre Montréal et Québec ou Toronto et New York. Et certains font l'aller-retour en Concorde en moins de 24 heures entre New York et Paris ou Londres pour négocier de visu - au siège social de l'autre centre-ville - au cour de la City, à Neuilly ou sur Wall Street. Certains auraient du mal à suivre la trace d'un Roben Lepage ou de quelques cadres. ingénieurs et techniciens de SNC-Lavalin entre plusieurs villes de deux ou trois continents ou... deux modestes et courtes pistes d'atterrissage de campagne pour rejoindre les monteurs de lignes envoyés par Hydro-Québec International.

\section{AU QUÉBEC}

Vu de Dorval, de Mirabel ou de SaintHubert, voire de l'aéroport Jean-Lesage (Québec), de Bagotville (aussi base militaire) ou de Val-d'Or (où peuvent atterrir des 747 (sic) déroutés par la mauvaise température au Sud), les milieux aéroportuaires d'ici sont modestes. Il y a plus de passagers (débarqués ou embarqués) à Chicago O'Hare en vingt minutes qu'à Mirabel en une journée... Mais, en 24 heures, au départ de Mirabel - bientôt (avril 1997) de Dorval -, les gens d'af- faires du Québec se retrouvent aisément en Afrique ou en Amérique du Sud, à Dakar ou Abidjan, Sao Paulo ou Buenos Aires. Par ailleurs, les gens d'affaires, d'associations et des reseatux venus de toutes les régions du Québec se réunissent souvent dans les salons du Hilton de Dorval ${ }^{16}$

Au cours des dernières années, au Canada. done au Québec. le systène aéroportuaire a connu et continue de connaître des changements majeurs : délégation de gestion à des autorités locales et régionales (Airport \&Defederalization*, selon un observateur attentif) ${ }^{17}$, privatisation du système de navigation aćtienne (en vigueur depuis le ler novembre $\left.1996^{15}\right)$, le tout dans un contexte de libéralisation du ciel (aOpen Skies en français du Québec) pout faciliter les déplacements professionnels entre Américains, Canadiens et Québécois, La situation nouvelle ne fait pas que des heureux: les transporteurs aériens srefu= sento d'acquitter la facture trop salée annoncếe pour fins de rénovation des aéroports ${ }^{(10)}$.

Et pourtant, chaque ville-région, plus que jamais, va devoir, concurtence oblige, investir avec ses partenaires dans l'amélioration de ses installations acroportuaires et, partant, dans $1^{+}$accessibilite (intermodale) aux aérogares ${ }^{\text {(30! }}$.

Les gens d'affaires voyageant sur une base quotidienne, veulent pouvoir arriver ă l'aéroport sans délai, se stationner facilement et, voyageant en classe affaires, s'enregistrer rapidement, profiter des nombreux services d"un salon particulier", faire un saut aux boutiques hors-taxes (vols transfrontaliess et internationaux) et... attendre que l'embarquement soit annoncér", puis, que l'appareil se rende en bout de piste et que le pilote ait enfin obtenu l'autorisation de décoller. Vive Dorval et Mirabel pour la faible densite...!

Aéroports de Montreal (ADM), qui jon= gle avec deux aeroports (en attendant de prendre en charge celui de Saint-Hubert) a. depuis les débuts (en 1992), mis les bouchées... doubles avee de très nombreux travaux, à Dorval principalement, (réaménagement de la jetée transfrontaliète, construction d'espaces commer- 
ciaux, nouveau hall d'arrivé, etc.) avec une nouvelle ab́rogare au centre-ville (depuis mars 1995) reliéc à la gare Centrale et au métro, des navettes ADM Express (autocar's Connaisseur), un service Valet à la carte à Dorval (cartes de crédit acceptées!). Dorval modernise son espace, un nouveau visage se dessine... ${ }^{12}$

Comme tout aéroport international, Dorval et Mirabel offrent une gamme de services (restaurants, location de voitures, stationnement, bureaux de change, boutiques hors-taxes, manutention des bagages, etc.). Mais..

Il y aura toujours, dans une catégorie ou une autre, un meilleur aéroport que celui où le voyageur va. Qui a acheté des lainages à Shannon (Irlande) ou des appareils électroniques japonais do Manaus (le long de 1"Amazone brésilien) peut éviter le casino de Schiphol (Amsterdam) pour avoir le temps de choisir un fromage Gouda mais ne pourra échapper aux contrôles de securité aux aéroports, de Munich à Tel Aviv.

En contrepartie, ADM aurait avantage à s'inspirer des nouvelles technologies déjà appliquées ailleurs comme, par exemple, le systeme informatique de concordance des bagages 2 ?

ADM ne peut évidemment rivaliser avec les plus grands. Le chroniqueur Normand Cazelais a déjà (décembre 1994) décrit, pour les lecteurs du journal Le Devoir, l'aéroport Charles-de-Gaulle (sous la responsabilite d'ADP, conme Orly) avec son amodule d'échanges* et sa gare TGV-RER à cing niveaux, doté d'un hotel grand luxe ${ }^{24+}$. Paris, une des dix principales places d'affaires mondiales...

ADM pourait se mesurer avec les neilleurs de sa catégorie. Encore ici, Normand Cazelais y va d'un exemple fort intéressant souvent cité, celui de l'aéroport SkyCity de Stockholm, ville d'une taille comparable à Montréal. Citation rapportée par le chroniqueur : * Cela ne dérange plus les voyageurs et gens d'affaires de ne pas aller à Stockholm, dans la mesure où ils trouvent ici ce dont ils ont besoin $^{(25)}$, w Sic !

Les aéroports de l'an 2000 ? Les inages de science-fiction de notre enfance sont en train de devenir réalité avec ces projets futuristes qui surgissent de l'eau à Hong
Kong, Bangkok, Séoul ou Tokyo,.. Comme le constate l'éditeur asiatique (qui est britannique) du magazine Flight International, l'Asie voit grand!'s Des projets d'aéroports qui ont de quoi dérouter les Occidentaux - et les Montréalais - par leur umpleur visionnaire. Souvenez-vous du périmètre original de... Mirabel, notre aéroport du troisième millénaire (vers 2015-2020) qui sera alors relié par TGV (2025) à la métropole américaine pour désengorger New York ${ }^{[27}$.

\section{SKY IS THE LIMIT...}

La très grande majorité des lecteurs (majoritairement détenteur's d'un diplôme universitaire) ont, à moins d'une peur maladive du mal de l'air, vraisemblablement emprunté l'avion plus d'une fois pour un déplacement professionnel quelconcue, comme un ATR 42 d'Inter Canadien, un DASH d'Air Alliance, un Boeing $(727,737,747,757,767)$, un DC-8 $8^{\text {on }}$ un DC-9 , un Airbus $\left(A 319^{\circ}, A 320^{\circ}, A 330, A 340\right)$, voire un L1011 de Royal Aviation ou d'Air Transat pour un colloque à Paris ou un palabre ensoleillé dans le Sud.

On aura depuis longtemps compris (ou percu) que des combats féroces sont depuis longtemps engagés pour la maîtrise d'un coin du ciel quand ce n'est pas le contrôle des comptoirs d'enregistrement et des salles d"embarquement (aslotss) aux aérogares.

Evidemment, il n'est pas donné (ou offert) à tout le monde d'avoir à se déplacer pour affaires, pour un congrès à Bali ou Delhi, pour une mission à l'île Maurice (quel sacrifice pour la francophonie !), pour un voyage d'études dans les iles grecques ou le long du Nil, pour un voyage de motivation (dit de stimulation ou vice-versa, ou incentive comme on dit à Marseille) à San Francisco ou San Antonio, pour un tour du monde (en trois semaines) pour le compte de (qui d'autres ?) SNC-Lavalin, pour une rénion de deux jours à Paris (soirée au choix) ou vancouver (joumée additionnelle à Whistler/Blackcomb). Que le tourisme d'aftaires est un tourisme diffus ! Il arrive assez rarement que les rencontres d'affaires aient lieu à Little Rock ou Winnipeg... (les nuits sont longues!). Même deux semạines ầ Atlanta pour les Jeux ne sont pas une sinécure.
Parlez-en aux globe-trotters du Service des sports de la SRC !

Yotre avion a pris son envol. Vos voisins de rangée ont-ils payé plein tarif ? ou obtenu un surclassement ? ou « gagné * un voyage d'agrément gratuit grâce au cumul des points-bonis des déplacements professionnels des six derniers mois (aux frais de l'entreprise ou de l'association ?) Champagne pour tous! (en Première ou en classe Affaires) avant d'incliner le quasi plantureux fauteuil - avec un empattement entre les sieges (avant et arrière) que les habitués d'Air Sardines (Transat, Royal et autres Corsair) ne sauraient imaginer - et de bien s'installer devant l'ecran individuel pour' profiter d'un choix de jeux, de lilms, de programmes de musique. Aux autres, en éco, de se voir offrir un plateau-repas digne de l'ancien Quebecair.

A moins que vous ne vouliez épater quelques collègues ou amis au bout du fil virtuel en glissant dans la conversation (entre deux gorgées de vin blane mousseux) que vous survolez actuellement Cleveland ou Gander... en route ${ }^{31}$ pour Houston ou Londres, Vous allez ouvrir votre micro portable pour une, deux, trois, six ou douze heures at l'abri du telephone, de l'assistant. des créanciers... Les avions, entre Paris et Montréal, Montréal et Vancouver, Vancouver et Tokyo, Tokyo et Paris, les plus merveilleux espaces de travail avec cinemabar bien approvisionne, restautant de qualitế, boutique hors-taxe et. règle générale, agents de bord attentionnés, compétents (sécurité oblige) ${ }^{35}$.

A toutes les echelles = vols internationaux, transfrontaliers, régionaux et locaux (sur la Basse-Côte-Nord par exemple), il y a des problématiques et des enjeux que, malheureusement, on enseigne peu aux jeunes inscrits à un DEC ou un baccalauréat en tourisme. Et pourtant les investissements des entreprises et des gouvernements, comme les dépenses des voyageurs, surtout d'affaires, en matière de transport aérien sont des plus considérables, voire, a l'occasion exhorbitants.

Dans le cas des concepteurs constructeurs, on travaille sur des échéanciers détaillés pour la période 2000-2025 (et mêne 2035) pour faire voler en 2015 jusqu'en 2040 les avions de l'avenit... immédiat. 
Déjà, chaque année, voit le jour (et vole!) des appareils de plus en plus sophistiqués, performants et de plus en plus rentables ${ }^{\text {toi }}$.

Here Come The Sky Giantsw titrait récemment le magazine Time ${ }^{37}$ et illustrait l'article des prochains autobus volants: Airbus $\mathrm{A} 3 \mathrm{XX}$ de 555 sieges (deux niveaux de passagers) et Boeing 747 $600 \mathrm{X}$ (548 sièges). Ces avions vont prendre l'air au tournant du siècle (et des poussières) et sont tres attendus (entre Tokyo et Sapporo, Hong Kong et Chica" go, etc.).

Le $A 3 X X$ va afficher une nouvelle dimension du confort pour les passagers: des salles de séjour et des chambres réduiront la fatigue due aux vols long courrier $^{281}$.

Selon les experts, plus de 1000 avions de ce type seront nécessaires d'ici à l'an 2000 ..

Les enjeux, comme les batailles, sont bien différents selon qu'on travaille chez un constructeur ou un transporteur. Les perspectives ne sont pas les mêtmes en termes de planification et de gestion: horizon 2005 vs horizon 2015.

Le cadre en marketing et ventes chez Boeing vend des avions (et les pièces de rechange - opération fort rentable), celui ou celle d'Air Canada propose ses vols et des sièges. Le premier se bat finement mais agressivement à un très haut niveau d'influence et de décision pour des budgets faramineux: un simple DASH 8-200 (37 sièges) est vendu, selon l'aménagement et l'habillement, entre 16 et 18 millions. Le second cherche à rejoindre (ou à conserver) les passager's les plus payants (les gens d'affaires) qui voyagent souvent et qui ont les moyens (ou ceux de l'employeur) de profiter des sièges de la Première ou de la classe Affaires: d'où les mille et une tactiques et techniques, des points-bonis ${ }^{39}$ au mille parcouru et un service à terre comme en l"air (voir illustration Air France), pendant que les voyageurs pensent faire du kilométrage avec leurs Air Miles (ou autres) par le biais de la fidélisation ${ }^{(+1) !}$.

Le transport aérien pourrait être un sụjet d'étude plus développé, notamment à Montréal, compte tenu de la vitalité du domaine aéronautique québécois, des sièges de l'OACI et de l'IATA. D'un point de vue plus systémique, l'univers des avions, jouets d'adultes, est un monde riche d'exemples d'études de cas: intégration verticale comme horizontale, grands groupes constitués par voie de fusions successives (amergers $\%$ ), partenariats politiques ou bconomiques, participations croisées ${ }^{i+1 !}$.

A toutes les échelles, internationales avec les très grands transporteurs qui sillonnent la planète continentale avec l'arrimage avec leur(s) transporteur(s) régional(aux) mieux connus sous le vocable de *feeders. Air Alliance et Inter Canadien jouent ce rôle au Québec pour Air Canada et... Canadien (pour le temps qu'il reste en vie (souffle court pour' combien de temps?).

A l'échelle locale, desserte des régions du Québec, de Mont-Joli à Bonaventure par exemple, il faudrait également souhaiter que le ministère du Transport et Tourisme Québec se donnent vraiment la peine d'inscrire quelques petites questions dans les mandats aux consultants pour qu'ils puissent commencer à s'interroger sur les liens possibles entre transport et tourisme, y compris le tourisme d'affaires.

De période de transition en période de transition (selon les gourous du management, des rationalisations et des exercices de planification stratégique), les tenants de l'économie IKEA du transport aérien continuent de courir pour arrimer offre et demande de tous les segments de micromarchés (géographiques, démographiques, etc.).

L'avion du XXle siècle, TGV des airs, va mettre à la portée de quelques milliards d'habitants d'Asie et du... Québec les 12000 pistes d'atterrissage du village global.

\section{CONCLUSION}

Annuellement, se brassent des centaines de milliards dans l'aeronautique à l"échelle mondiale"t2y , Quand allons-nous voler sur un supersonique gros porteur? 2025? 2035? 2045'? Et pour quelles destinations?

En fait, l'avenir est indéchiffrable comme toujours, mais l'idếe qu'on s'en fait commande l'état d'esprit du présent et devient du même coup un élément $\operatorname{actif}^{2+31}$.

Comme l'a déjà souligné Bernard Marck: À quel Saint (Ex) se vouer?sit4, It's a flat world after all... La fin du travail - à l'êre de l'économie IKEA - ctant inexorablement, pour plusieurs travailleurs dans la production, va favoriser l'expansion des voyages (mobilité professionnelle, temps libre, culture du nomadisme du XXIe siecle, etc.).

Bienvenue au XXIe siècle! Le futur est défà arrivé. Même le unouveaù Journal de Montréal présente maintenant une météo spécialement conçue pour les besoins des gens d'affaires qui voyagent. Pierre Péladeau, du haut de son hélicoptère personnel, qui, matin et soir, emprunte la voie des airs entre Sainte-Adèle et le centre-ville de Montréal, a, comme Laurent Beaudoin (qui, lui, vole - quasi quotidiennement - sur un Challenger ou sur un... Learjet), compris $1^{+}$importance stratégique de l'aéronautique dans le développement des industries du tourisme et du voyage, non seulement aux plans international et continental mais aussi pour l'avenir des régions du Québec.

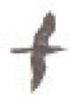

\section{NOTES}

(1) A titre d'exemple, le Hilton (The Hilton comme on dir à Paris) de Roissy Charlesde-Gaulle, ouvert en 1994, e'est 20 salles de conférences, une salle de réception de 900 personnes, des prises pour transmuission informatique dans les. 383 chambres, un fitness club (en français de France). Bref, comme le souligne leur message publicitaite, wun grand hôtel et un grand centre d'affaires .... à deux pas du RER.

(2) NAISBITT: John (1994), Ghohol Parador. p. 189.

(3) Pour mémoire, plus de $21 \%$ de la population mondiale vit en Chine.

(4) Le modèle dernier cri (décembre 1996) est, selon FORTLNE. Travelpro Businesstius Rollaboard (23 décembre 1996), p. 258, 229,95 S US : * this particular model has a padded laptop case hidden inside that zips open separately so you can get at your computer without disturbing the rest of your bag. It even provides a handy fold-down desk/shelf, should you be unlucky enough to wind up stuck at $\rightarrow$ (the airport). N.B. : la majorité des gens d'affaires ont, bien sür. des produits American Tourister, Lark, Samsonite, Jaguar, etc. 


\section{AVIONS D'AFFAIRES}

Observée dans l'environnement nord-américain, la bataille entre fabricants de jets d'affaires se limite à deux agrands: Canadair (Bombardier) avec le Challenger (surtout le CL 604 ) et, bientót, le Global Express et Learjet (Bombardien) surtout avec le Learjet 60 d'une part, et Guffstream avec le IV.SP et. tres bientot le $V$ d'autre part. Cessna, avec les Citation ou Astra (Israël Aircraft Industries) avec les Galaxy occupent des créneaux. Et ce, sans compter les nombreux constructeurs de turbopropulseurs.

Vue a l'échelle mondiale, il faut compter avec d'autres ngrands", au premier chef. Dassault et les Falcon $900 \mathrm{~B}$ ot 2000 (certains "petits" avions d'affaires sont des trireacteurs),

La National Business Aircraft Association (NBAA), lors de sa convention annuelle (la 49 e en $1996 \mathrm{D}$, présente une exposition de quelque 200 avions differents. Plusieurs entreprises - souvent multinationales - les gouvernements et les princes ont leur propres flottes de six, huit, dix jets.

Flexibilité, vitesse, service à la demande sont les atouts de l'aviation d'affaires pour des coûts parfois très... compétitifs. Nombreux sont coux qui, depuis longtemps, ont démystifíá le jet d'affaires au-delá de l'image valorisante ${ }^{45 i}$.

\section{MONTRÉAL AÉRONAUTIQUUE}

Récemment (début novembre 1996), les médias québécois nous apprenaient que Boeing faissant des démarches à Montréal pour embaucher des ingénieurs qualifies et experimentés.

Un dossier spécial de l'hebdomadaire Les Affaires (13 juillet 1996 ) parle d'une sindustrie qui roule à plein régimes. Trente cinq mille emplois et sept cents postes a... combler. En 1994, Cdossier spécial du 9 juillev, Les Affaires sinterrogeait $* Y$ aurait-il une vie sans Bombardier?.

CAE Électronique, Hóroux, Mecair, Pratt \& Whitney, Bell Helicoptère Textron, Air Canada, Marconi, etc... L'incertitude politique? Quelle incertitude? II est normal qu'il $y$ ait des turbulences dans le domaine aéronautique!

\section{BOMBARDIER}

C'est une societé canadienne (siege social à Montreab exerçant des activites de conception, de développement, de fabrication et de commercialisation dans trois domaines: matériel de transport, produits de consommation motorisés et.. aéronautique let services reliás) qui exploite des usines dans neuf pays et emploie 40000 personnes. Chiffre d'affaires de Fexercice clos le 31 janvier 1996: 7,1 milliards $\$ C N$.

Le Groupe aéronautique réunit toutes les activités aéronautiques tant en Amérique du Nord quien Europe, soit Canadair et de Havilland au Canada, Learjet aux États-Unis de même que la division aéronautique de Shorts en Irlande du Nord. II faut reconnaitre que Bombardier s'est taillé une place de chef de file dans le marché des avions d'affaires et de transport régional ${ }^{\text {atu. }}$.

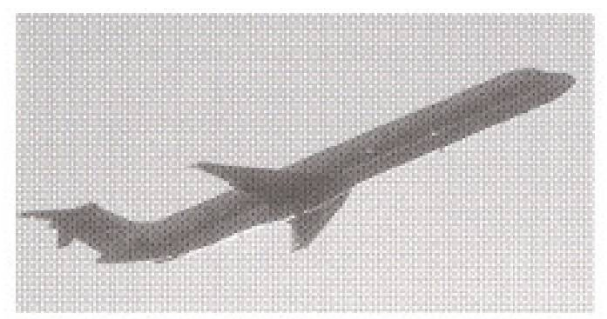

\section{AIR CANADA - CANADIAN}

a) Hollis Harris has led Air Canada out of the financial wildesness. After 4 years of losses, the airline is profitable and grow. ing*.

FLINT, Perry (juin 1995), *The Business is actually free again !s, Air Transport World, Washington, p. 26.

b) If they're (Canadian Airlines International) successfull in getting the co-operation that they're requesting... then the chances of the company continuing to operate as it is currently configured would appear to be assured for the near term.

LARKIN, Ted canalyste de la firme Bunting Warburg lnc), (10 décembre 1996). The Financial Post, p. 3.

Comme on dit au Canada, the show must go on ! A suivre... de près par analystes financiers interprosés. Le meilleur expert est le Quebecois Jacques Kavafian (Research Capital Corp. à Torontol souvent cité dans les pages économiques des quotidiens.

\section{AIRBUS ET [vs] BOEING}

L'Europe est depuis plus de quinze ans à la poursuite de l'Amerique. Pendant l'aventure (echec commercial) franco-britannique du Concorde, Air France et British Airways emportaient et emportent encore leurs passagers sur des... boeings (majorité des flottes).

Le consortium Aérospatiale (France), DAZA (Allemagne), British Aerospace et CASA (Espagne) avait réussi à gagner du terrain. dépassant avec plaisir le numéro deux de l'aéronautique, McDonnell Douglas, qui annonce, ce 15 décembre, qu'il devient par: tie de Boeing pour creer le plus gros avionneur mondial. Lapprobation des autorites de Washington sera vraisemblablement une formalité (en 1997).

Difficile de mesurer tous les impacts faute de recul. II ne faut pas perdre de vue que Boeing et McDonnell Douglas travaillent beaucoup dans les domaines de la défense et de l'aérospatial. Et les deux entreprises ont des filiales au Canada. N.B.t Bombardier devient le troisième concepteur et constructour d'avions au monde... mais se spécialise dans les appareils régionaux et les jets d'affaires:

\section{AÉROPORTS DE MONTRÉAL}

ADM est une administration aéroportuaire locale (AAL responsable de la gestion et du développement des aéroports de Montróal par l'entremise d'un bail d'une durce de 60 ans signé avec Transports Canada. Le gou= vernement fédéral conserve la juridiction des douanes et de la securité (GRC) sur les sites. La gestion des services aèroportuaires incombe entierement à $\mathrm{ADM}$. ADM est une société à but non lucratif... avec nombreuses filiales...

C'est le conseil d'administration de la Société de promotion des aéroports de Montréal (SOPRAM) composé de 21 membres nommés par les organismes du milieu qui agit aussi comme conseil d'administration d'ADM Ville de Montréal, Ville de Laval, Conférence des maires de banlieue, Chambre de commerce du Montréal métropolitain, Corporation de promotion à Mirabel (COPAM) et Société montérégienne de développement (SMD) et Ville de Longueuil, conjointement. 
(5) LATOUCHE Daniel (1990) wLes violons tristesw, Le Devoir, 12 janvier.

(6) MARTIN, Fernand et MARTIN, Robert (1996). Hawnonisation des donnés meiropolitaines Canada-Etats-Unis avec reference a la région métropolitane de Montrial. Ville de Montréal, 53 pages.

(7) Les Etats-Unis et I'Union européenne ont entamé au cours de l'automne 1996 leur premièré serie de discussions pour établir des a regles equitables de libre concurrence sur I'Atlantique Nord et ceventuellement, avec d'autres pays woisins comme ceux

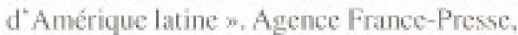
29 octobre 1996

(8) En novembre 1996. USAIR et Airbus ont conclu une vente de 120 avions movencourrier acoompagnée d'une option portant sur 280 appareils. Au cours du même mois, American Airlines ef Boeing ont annoncé une entente de longue duré (1998-2018) de l'ordre de 35 milliards \$ (US) pour la livraison de 630 (sic) appareils ( 103 commandes fermes et 527 options pour des 737,757 , $767 \mathrm{et} 777$. II faut etre très prudent avee de telles options et la valse des milliards avant de conclure que les 900 avions seront bel et bien livrés. Les constructeurs comme les compagnies atriennes peuvent avoir des motifs stratégiques a faire des effets d'annonce. On l'a déjà vu avec l'annonce du 15 decembre 1996 (fusion Boeing-McDonnell? Qui vivra verta ! Le 747 , comme le supersonique Concorde, auront-ils êté des avions du... deuxième millenaire?

(9) L'Association du transport aérien international (IATA) compte actuellement 256 transporteurs membres représentant 70 pays. En 1905, ils ont transporté 352 millions de passagers. Le siège d'IATA comme celui de l'OACl - Organisation de l'aviation civile internationalel est a Montréal. Le directeur géneral et l'orgas nisme est l'ancien president directeut génétal d'Air Canada, Pierre J Jeanniot; il est aussi chancelier de I'UQAM... II vient d"annoner ( 16 décembre 1996) une accentuation de l'implantation d'TATA dans la métropole québésoise en transférant certaines sctivités majeures de Genève à Montréal. Quand on prend dé lá hauteur, les debats constitutionnels locaux apparaissent moins... gros (porteurs). N.B.: Messieurs Chrétien et Bonchand ont participé le 5 decombre 1996 a l'inauguration de la Maison de l'OACl (183 pays membres) construite au cocur de la cité internationale de Montreal aul coût de 100 millions. L'édifice de quinze étages, à proximité du Palais des congrés, de Place Bonaventure et du Centre de commeree mondial, accueille 800 employés

(10) Voir MARCK, Bernard (1995), wChantiers aéroportuaites: un avenir charges, Aerwpors Magazine, no 255. p. 20-41.
(11) Laéroport Chep Luk Kok (coût estimé ì 21 milliands \$) poumb, at teme, trater une capacite de 87 millions de passagers.

(12) Plus de $50 \%$ de la population mondiale y vit. Qui plus est, il s'agit de communutes jeunes.

(13) On estime à près de 250 millions de personnes le nombre de citoyens indiens qui composent la classe dite moyenne.

(14) Des sommes considerables ont été Egalement investies a l'atroport Pearson (Toronto) pour moderniser les installations existantes (Terminals I et 2) apres l'ouverture (cn fövier 1991) du magnifique Trillium Terminal III. Ce derniner, construit et géré par le secteur privé, a presque tout pour plaire aux plus exigeants: with 100,00 sque feet of foodservice and retail space, travellers will now be able to choose from at plethora of different foodservice experiences, from fast-food to more elegant dining. And for weary travellers who don't want to travel far and wide to get to their hotel rooms, Swissotel introduces its first Canadian property, conveniently connected by a skywalk to the terminal. Finally, for those travellers who like to "shop til they drop", Terminal III is teeming with upscale retail outlets including the workedrenowned Harrod's of London_.5 in Food. service and hospitalisy Report, June 1991, p. 33.

(15) Au cours des douze demieres années, il y at eu au moins trois tentatives d'assurer des Iialsons directes (sans escale) entre Québec et la Grosse Pomme. II faudrait sérieusement s'y meltre avec une stratégié marketing au moins biennale affaires-agrement (types d'appareils, horaires, forfaits et promation).

(16) aRendez-vous à l'ateroport. Lequel, au vôttre ou au mien ?\% Boutade rapportéce par CAZELAIS, Normand (1996) wLes aeroports de l"avenirs, Le Devoir, 5 février. Dans sa chronique, l'auteur signalait un article rếcent publié par le Intematronal Herold Tribure intitule a Ainports of Fuare Ship Cilies

(17) HAMILTON, Gordon (1994), airport "Defederalization" - A New Industry in Canadar, ATAC Bulletio, mai, p. 3. Extrait: Canada is one of the few developed nations in which the federal govermment directly manages the airports. One result of this has been national standards that do not reflect local market conditions...

(18) L'ensemble du réseau, et les 6000 emplois fédéraus affectes, ont été transferés de Transports Canada a NAV Canada, pour une contrepartie de 1.5 milliard. Selon la Presse Canadienne, les activités de la nouvelle entreprise seront financees par des frais aux usagers que percevront les compagnies acriennes, Controble privé dans les tours: Le Dewoir, ler nowembre 1906 . N.B. : Le plus important appel public a l'epargne dans I'histoire du Canada sest terminte le 16 décembre 1996 par la clature d'une émission de 750 millions d'obligations-recettes (arevenue bondsw) effectué par Nav Canada. Lappel public a été entierement souscrit. Nav Canada a vait ếmis ees obligations dans le but de rembourser une partic de sa dette de trois milliards contracté le 31 octobre dernier pour financer l'acquisition du systeme de navigation aérienne civile du gouvemement féderal. Woir La Presse. 17 décembre 1996

(19) Affrontement entre directeurs des aéroports et representants des compagnies atricnnes, lors de l'assemblec annuelle de l'Association canadienne du transport aérien (ATAC), quand uété abordée la question du paiement de quelque quatre milliards de rénovation maintenant que le rédéral a decide de ne plus faire ces investissements. Woir Le Devoir, 19 novembre 1996.

(20) Dans le sillage de la décision d'ADM de ramener tous les vols internationaux réguliers de Mirabel is Dorval à compter d'avril 1997. Ie ministere québeois du Transport s'est vu obligé de revoir ses priorites dans le Grand Montréul. Au Sommet sur l'économie et l'emploi (oct.-nov. 1996), ont êt antioness des investissements de 36 millions \$ pour améliorer les voies d'acces il I'aénoport de Dorval (Groupe de trawail sur la relance de la métropole).

(21) Ceux et celles qui ont un tant soit peu voyagé (eu la chance de) aux frais de la princesse ou de l"entreprise peuvent debattre des mérites des aéroports et des espaces que les compagnies aériemes y aménagent. Pour les epauvress qui n'ont pas les moyens d'empeunter le Concorde ou de wolers en Premiere, l'auteur suggere la Upper Class (1 0005 de moins que la First réguliere) de Virgin (la compagnie de Richard Branson): au Virgin Clubhouse de Heathrow, on $y$ reçoit massage, facial et manucure en doucecur... N.I. : pour éviter la circulation Iondonnienne, si vous voyagez léger, Virgin iral vous chercher en moto plutŏt qu'en limo! Voir at ce sujet TEITELBAUM. RICHARD (1996), My Airpor, My Palace in Fortune, 22 juillet, p. 152-153

(22) Espace. Sera publie quatre fois par année par les Services à la clientèle et le service des Affaires publiques et communication d'ADM. Vol. 1 -Octobre 1996, 8 pages (anglais-français).

(23) Voir PAYNE, Robert A. (1995), Le système informatique de concordance des bagages se caractérise par sa süreté optimale et sit commoditê Journal OACl, janvier/fevrier, p. 20-21. Le systeme en vigueur à Francfort depuis 1993 est branche atux ordinateurs centraux de plusieurs transporteurs ateriens.

(24) CAZELAIS, Normand (1994), wCharlesde-Gaulle : une aérogare internationale $L A$ Dewoir, 19 decembre, p. 3.

(25) Article deja cite, note 16, Le Dewor, 5 fevrier 1996. 
(26) Voir LEWIS, Paul (1996), w Thinking Big, Building for the Futures, Swissair + Gazelte, vol. 4 no 26, p. 54-56.

(27) Ceux et celles qui auraient un peu de mal à partager cette hypothèse seront heurcux d'apprendre qu'atu début de décembre (1996). les autorités municipales de Las Angeles (LAX en langage ateroportuaire) ont dévoilế un plan directeur pour améliorer l'aéroport principal de ecte porte ouverte sur l'Asie. Le smaster plans prévoit des investissements pouvant s'élever à 12 milliards (US, bien sûr) pour pouvoir recevoir 93 millions de passagers en 2015 , une augmentation de $60 \%$ par rapport aux données actuclles.

(28) Titre cmprunté à l'auteur. PELLETIER. Jean (1900), "La deréglemetation du transport aérien aux Etats-Unis: $\alpha$ The sky is the limit.. "s, Teoros, vol. 9, no 2, juillet, p. 4336, et PELLETIER, Jean (1984), whe place au ciel pour tout le mondes, Teoros, vol. 3, no 2, juillet, p. 11-14 et 19-20.

(29) Le DC-8 étuit une des spécialités de feu Nationair fcrée en 1984, pilotée par Robert Obadia qui, avec sa femme et son fils, fait face ì nombre de chefs d'accusations en vertu de la Loi sur les faillites. Proces fixé au 16 juin 1997.

(30) Le transporteur Air Canada a, au cours de l'automne dernier, annoncé que, pour' faire face à une croissance soutenue, il doit finalement conserver 15 des 35 vicux DC-9 (92 passagers) jusqu'en 1998-1999 (minimalement) en y mettant le prix avec unc mise aux normes (5 millions par appareil), entre autres pour ajouter les fameux silencieux $(*$ hush kits $x$ ) au cout de 2,5 millions \$ par DC-9

(31) Ail' Canada a reçu en décembre 1996 son premier $A 319$ (112 passagers), d'une série de 35 appareils, qui arrivent au rythme de deux par mois a compter de janvier. Air Canada est le premier transporteur nordaméricain ì faire voler ce type d'avion du nord au sud et d'est en ouest. Ce sera le nerf air canadien (avec, évidemment, les $\mathrm{RJ}$ de Canadair) pour livrer la bataille des cieux ouverts.

(32) Air Canada compte déa depuis quelques années des A320 el A340 (long courrier)... Dossier complexe. Suite particlle à venir dans le cadre des débats devant les tribunaux où s'opposent les avocats de Brian Mulroncy et les procureurs du gouvernement fédéral.

(33) Voir à ce sujet un numéro spécial collection Les cahiers d'Espaces, Le Tourisne d'Affaires, no 10, juin 1988, 80 pages.

(34) EnRoute. Revue de bord mensuelle gratuite sur Air Canadat qui présente mille et une propositions aux gens d'uffaires (celles d'AT\&T, Sharp, Sprint Canadi, Canada direct Téléglobe). American Express, quelques suggestions culturelles el gastronomiques (a destination) sans oublier, bien sûr les produits Vacances Air Canada el... la carte Diners Club International EnRoute. Chaque transporteur aérien at b́videmment son périodique sus papier glace présentant souwent les mêmes annonceurs (cartes de crédit, location de voitures, etc.).

(35) Voir HAILE, Belaii (1996), «Les agents de cabine sont un element important de la sécurité dans la prévention des accidents et dans l'assistance aux passagersw, Journal $O A C l$, juin, p. $10-11$ et 29 .

(36) BOUEY, Jim (1996), "Les nouvelles technologies des cellules, des systemes et des moteurs ameliorent les performances des triréacteurs: Journal $\mathrm{OACl}$, janvier-fésvrier, p. 7-9 et 24 .

(37) 16 septembre 1996, p. 31-32

(38) LAMBY, Stephan (1996), "L'A3XXX; le futur membre de la famille Airbuss, Deutschland Magazise, no 4, aouit, p. 34-35.

(39) Le dossier des apoints mériterait à lui seul un dossier Terors. Les institutions financières (bangues et apparentées comme AMEX) avec leurs VISA et MASTERCARD, les transporteurs principalement aćriens) et les chânes hôtelières ont imaginé des sprivilègess qui finissent par conditionner certains voyageurs d'affaires it organiser leurs itineraires de façon à leur garantir la gratuité des billets d'avion pour les vacanoes annuelles de toute la famille... à l'autre bout du monde. Ah les « frequentthiers:!

(40) Voir CITRONOT, Luc (1994), aFrequent Flyer, le prix de la fidélités, Vovages d'Affores, no 28, mars-avril, p. 27-38, II faut savoir jouer ses... cartes!

(41) Dans l"industrie du transport, comme dans celles du tourisme et du voyage, on peut observer depuis 25 ans les tendances structurelles (globalisation et actions locales ou cibleses). On voit, par exemple, la filiale no 126 de la multinationale $\mathrm{A}$ s"associer à la filiale no 77 de la multinationale $C$ pout onvalero la filiale no 28 de la multinationale B. Pensons aux Lyonnaise ou Générale des eaux en France ou at... Boeing-McDonnell Douglas ou encore à... Bombardier et American Airlines dans la compangnie Business Jet Solutions pour louer des avions d'affaires en temps partagé.

(42) Un seul exemple; le système de compensation financière inter transporteurs d"IATA a abrasséw. Ajoutes l'aménagement des installations acroportuaires, la vente de quelques centaines d'avions (gros porteurs, avions regionaux, jets d'affaires).

(43) Voir GIROUX. Françoise (1996). Le Nowel Observaleur, 27 juin.

(44) MARCK, Bernard (1994), «Débarquement littéraire sur tous les fronts\%, Aeroports Magazine, no 250, juillet-aout. p. 54-55. Liduteur rappelle que le fondateur de I'Aéropostale avait prédit (cn 1944) le probable lendemain d'aprés-guerre de démocratisation du transport aérien civil où ules aviateurs deviendraient des conducteurs d'autobus volantss.
(45) NOYE Régis (1994), -Aviation d'affaires l'après-crise». Aéroports Magazine, no 253. novembre, p. 50-51 et CITRINOT, Luc (1992). aviation privéc; des affaires à l'incentives. Voyages d'affaires. no 19. mai-juin-juillet, p. 36-41.

(46) Au cours des cinq derniers mois de 1996. Bombardier a fait les (bonnes) nouvelles avec (entre autres) les annonces suivantes: a) 25 Dash 8-200 ( 37 passagers) vendus en août à Horizon Air (Seattle), filiale d'Alaska Air Group (N.B. : à ce jour, plus de 500 Dash 8 ont ćté livrés ou commandés), by ler vol experimental du Global Express le 13 octobre cpourra ameer huit passagers sur $12000 \mathrm{~km}$ à Mach 0,89 ); dejà plus de 50 commandes, el press de $200 \mathrm{RJ}$ (Regional Jet de 50 sieges - déjà fort utilisés par Air Canada (24 appareils) et Lufthansa - ont déjà été vendus, d) " prêt * de 87 millions \$ du gouvernement fétéral pour le RdD en vue du CRJX, version allongé (70 sièges) du bi-réacteur RJ, e) en novembre, annonce de la lo0e vente du Learjet 45 (different du $31 \mathrm{~A}$ ct du 60 ) : le premier appareil assenblé a été dévoilé aux (nombreux) intéressés le 14 septembre.. 1996 (sic); toute lá production prévuc jusqu'cn 1999 est dejà soldee aux riches d'Arabic et d'ailleurs et peu célèbres (sur la place publique), f) Bombardier achète (début décembre) la Division aménagement et finition d'avions d'aftaires INNOTECH pour l'habillage des Challenger et des Global Express et gy au moment de mettre sous presse le présent numéro de Tcoros, le gouvemement canadien était sur le point d'annoncer un nouveau * prêt \& (54 millions) pour la R\&D sur un nouveau Dash 8 400 (70 passagers). Bref. Bombardier fait flèche de tout bois. 